\title{
Dissection of Gain Control Mechanisms in Drosophila Mechanotransduction
}

\author{
Abhishek Chadha and Boaz Cook \\ Department of Cell Biology, The Scripps Research Institute, La Jolla, California 92037
}

Mechanoreceptor cells respond to a vast span of stimulus intensities, which they transduce into a limited response-range using a dynamic regulation of transduction gain. Weak stimuli are detected by enhancing the gain of responses through the process of active mechanical amplification. To preserve responsiveness, the gain of responses to prolonged activation is rapidly reduced through the process of adaptation. We investigated long-term processes of mechanotransduction gain control by studying responses from single mechanoreceptor neurons in Drosophila. We found that mechanical stimuli elicited a sustained reduction of gain that we termed long-term adaptation. Long-term adaptation and the adaptive decay of responses during stimuli had distinct kinetics and they were independently affected by manipulations of mechanotransduction. Therefore, long-term adaptation is not associated with the reduction of response gain during stimulation. Instead, the long-term adaptation suppressed canonical features of active amplification which were the high gain of weak stimuli and the spontaneous emission of noise. In addition, depressing amplification using energy deprivation recapitulated the effects of long-term adaptation. These data suggest that long-term adaptation is mediated by suppression of active amplification. Finally, the extent of long-term adaptation matched with cytoplasmic $\mathrm{Ca}^{2+}$ levels and dTrpA1-induced $\mathrm{Ca}^{2+}$ elevation elicited the effects of long-term adaptation. Our data suggest that mechanotransduction employs parallel adaptive mechanisms: while a rapid process exerts immediate gain reduction, long-term adjustments are achieved by attenuating active amplification. The slow adjustment of gain, manifest as diminished sensitivity, is associated with the accumulation of $\mathrm{Ca}^{2+}$.

\section{Introduction}

In order for mechanoreceptor cells to resolve magnitude differences, they accentuate deviations of stimuli from their background levels by matching the transduction gain with the average input intensity (Hudspeth and Gillespie, 1994). In a variety of mechanoreceptor cells, a sustained stimulus generates a large initial response that rapidly decays through the process of adaptation, reaching a diminished steady-state level (Eatock et al., 1987; Walker et al., 2000; O'Hagan et al., 2005). Typically, at the adapted state, sensitivities to further stimuli are retained (Eatock et al., 1987). In hair cells, adaptation is comprised of two separate calcium-dependent processes (Wu et al., 1999). One adaptation process is rapid and results in channel reclosure (Kennedy et al., 2003), and the other is a slower process which adjusts mechanical compliance during a stimulus (Howard and Hudspeth, 1987). Suggested underlying mechanisms for fast adaptation include direct binding of $\mathrm{Ca}^{2+}$ to the transduction channel (Crawford et al., 1991), relaxation of ankyrin subunits in series with the channel gate (Howard and Bechstedt, 2004) and conformational changes in channel-associated myosin motors (Stauffer et al., 2005). For slow adaptation, tension adjustment through myosin

\footnotetext{
Received May 5, 2012; revised June 19, 2012; accepted July 15, 2012.

Author contributions: A.C. and B.C. designed research; A.C. and B.C. performed research; A.C. analyzed data; A.C. and B.C. wrote the paper.

We thank Kathryn Spencer for technical support. We thank Tomer Avidor-Reiss, Maki Kaneko, and Sung Eun Kim for comments on an earlier draft of the manuscript.

Correspondence should be addressed to Boaz Cook at the above address. E-mail: bcook@scripps.edu.

DOI:10.1523/JNEUROSCI.2171-12.2012

Copyright $\odot 2012$ the authors $\quad 0270-6474 / 12 / 3213052-10 \$ 15.00 / 0$
}

motors is the prevailing model (Holt et al., 2002; Gillespie and Muller, 2009); however, it has been challenged by recent results of imaging experiments (Beurg et al., 2009).

At the lower end of the intensity range, auditory thresholds are close to thermal noise levels (De Vries, 1948), reflecting the process of active mechanical amplification which increases mechanotransduction gain (Hudspeth, 2008). The amplification is termed active since it is a process that produces mechanical forces (Brass and Kemp, 1993) and it requires energy supply (Robertson and Manley, 1974). Single mechanoreceptor cells are thought to underlie active mechanical amplification since hair cells exhibit all of the manifestations of the process, including enhanced gain of minute inputs and the production of spontaneous mechanical emissions (Jaramillo et al., 1993; Martin and Hudspeth, 1999, 2001; Martin et al., 2003). These spontaneous mechanical emissions are accompanied by an electrical component (Crawford and Fettiplace, 1985; Martin et al., 2003).

Mechanical amplification and spontaneous mechanical emissions have also been demonstrated in the hearing organ of Drosophila (Göpfert et al., 2005, 2006). Functional mechanotransduction was required for both phenomena, indicating that as postulated in vertebrates, the transduction machinery in Drosophila is necessary for producing active amplification (Göpfert et al., 2006). Adaptation has also been characterized in Drosophila (Walker et al., 2000), making the flies well suited for studying the contribution of both active amplification and adaptation to gain control in mechanotransduction.

The role of gain-modulating transduction processes in long-term adjustment to activation is not clear. We found that 


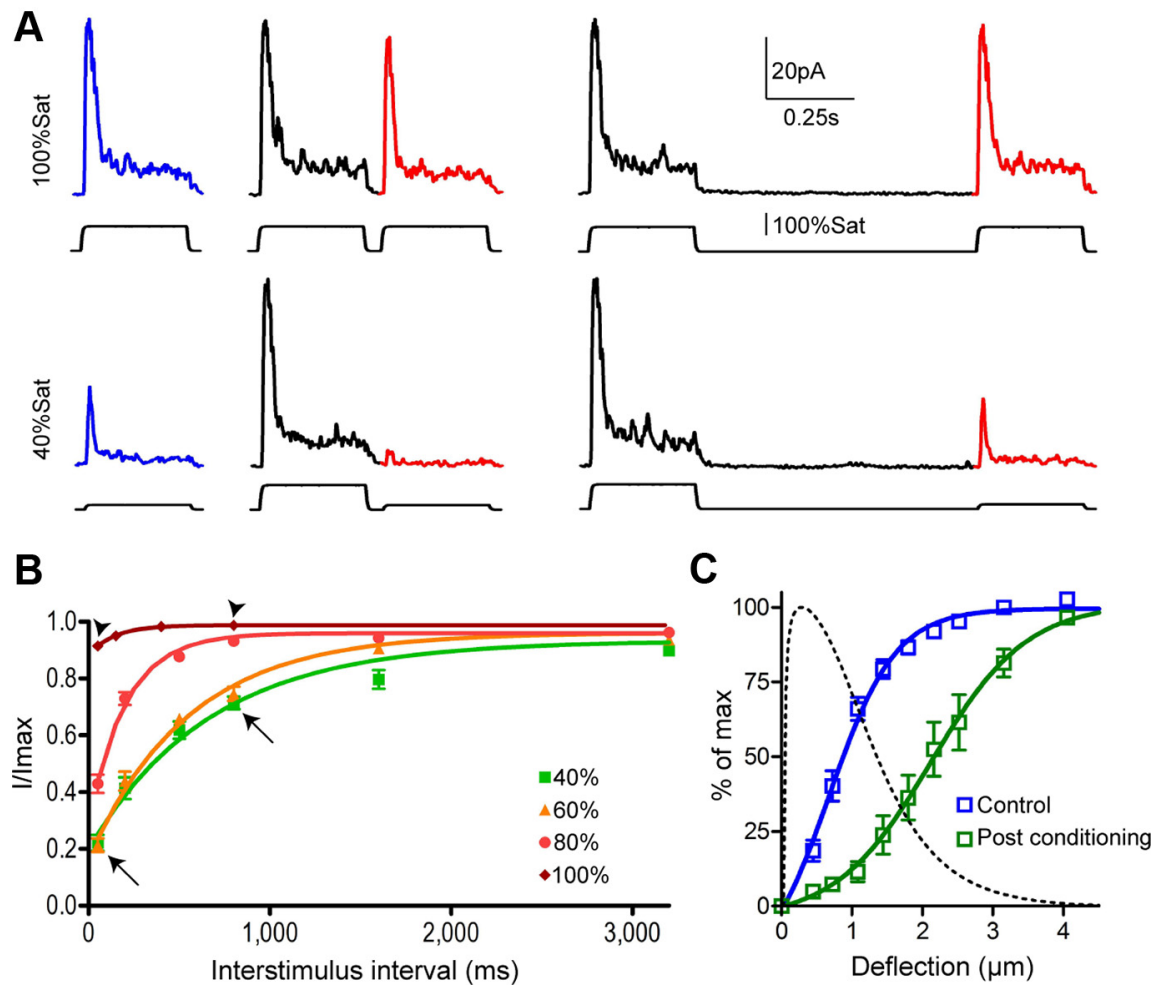

Figure 1. Characterization of activation-induced depression. $\boldsymbol{A}$, Current responses to mechanical stimuli showing test responses before (blue) and after (red) conditioning pulses (black). A 100\%Sat and a 40\%Sat test pulse are demonstrated, each at 50 $\mathrm{ms}$ (left) and $800 \mathrm{~ms}$ (right) interstimulus intervals. $\boldsymbol{B}$, Recovery of different test-pulse magnitudes fitted with monoexponential time courses. Arrowheads correspond to the top traces of $\boldsymbol{A}$ and arrows to the bottom traces. $n=5-8$. $C$, Normalized currentdisplacement curves before (blue) and $25 \mathrm{~ms}$ after (green) a $600 \mathrm{~ms}$ conditioning stimulus. The solid lines are Boltzmann fits and the dotted line is a normalized extent of suppression. $n \geq 6$.

in Drosophila mechanoreceptors, activation causes a reduction in sensitivity that lasts for, and develops during hundreds of milliseconds. Electrophysiological and genetic manipulations of mechanotransduction revealed that long-term adaptation is not associated with the response decay induced by adaptation. Instead, long-term adaptation is associated with suppression of active amplification. Finally, we provide indications that accumulation of $\mathrm{Ca}^{2+}$ plays a role in mediating long-term adaptation.

\section{Materials and Methods}

Fly stocks. We used the following stocks: $\mathrm{w}^{1118}$ were used as controls unless otherwise stated. UAS-dTrpA1/TM6B [Bloomington Drosophila Stock Center (BDSC), 26264], UAS-GCaMP3 (BDSC 32116), Cha-Gal4, nompC $C^{4}, \mathrm{cn}$ bw.

Electrophysiology. Recordings were performed on flies of either sex by mounting a glass pipette over a cut anteronotopleural bristle (Walker et al., 2000). For stimulation, the pipette was displaced using a PI E-625 piezoelectric system. The recording pipette contained, in mM: $121 \mathrm{~K}^{+}, 9$ $\mathrm{Na}^{+}, 0.5 \mathrm{Ca}^{2+}, 4 \mathrm{Mg}^{2+}, 35$ glucose, and 5 HEPES, pH 7.1, and the reference pipette contained $2 \mathrm{~K}^{+}, 128 \mathrm{Na}^{+}, 0.5 \mathrm{Ca}^{2+}, 4 \mathrm{Mg}^{2+}, 35$ glucose, and 5 HEPES, pH 7.1. Holding potential was $40 \mathrm{mV}$ unless otherwise stated.

Currents were sampled at $5 \mathrm{kHz}$ and filtered at $0.1 \mathrm{kHz}$ using a PC-501A amplifier (Warner Instruments). Unless otherwise stated, $85 \%$ Sat conditioning stimuli were used to extend the survival of the preparation.

A saturating stimulus was determined by applying a set of incrementally increasing stimuli that cover the response range. The first response that was not significantly enlarged ( $<5 \%$ increase from previous response) was defined as $100 \%$ Sat. All other response amplitudes recorded from the same receptor neuron were normalized to the $100 \%$ Sat value. The displacement that elicited the $100 \%$ Sat response is defined as the $100 \%$ Sat stimulus. When the independent variable was stimulus intensity, displacements of the same bristle were normalized to a $100 \%$ Sat stimulus.

Data analysis. The time constant of longterm adaptation onset was determined by fitting the relationship between conditioning stimulus time and normalized current recovery to a monoexponential decay. Comparisons between long-term adaptation onset and adaptive response decay were performed in the same flies, and the adaptive response decay time constant was determined by fitting the current relaxation to a monoexponential decay. The time constant of long-term adaptation persistence was determined by fitting the relationship between interstimulus intervals and normalized currents to a monoexponential association.

The full current-displacement curve was performed in flies that reached $100 \%$ Sat at 3.15 $\mu \mathrm{m}$. The conditioning stimulus was $3.15 \mu \mathrm{m}$ and responses were determined at displacements of $0.45,0.72,1.08,1.44,1.8,2.16,2.52$, 3.15 and $4.05 \mu \mathrm{m}$. Half-saturation point was determined by a Boltzmann fit to the current-displacement curve. Time constants for monoexponentials, $\mathrm{X}_{50}$ values for current-displacement curves, and slope values for current-displacement curves were determined in Prism 4.0 (GraphPad Software). The extent of suppression was determined by dividing the control Boltzmann fits by the shifted ones, and normalizing the resulting curve to its peak value.

Baseline variance for spontaneous noise experiments was determined using a moving variance with a window of 100 $\mathrm{ms}$. Variance was sampled every $10 \mathrm{~ms}$ and normalized to the variance of a $1 \mathrm{~s}$ segment of baseline before the response. Total charge was calculated by integrating the current elicited by the stimulus (picocoulombs/second) over the duration of the stimulus (seconds), resulting in units of charge (picocoulombs). A custom Java script was used for automated determination of peaks, integration of total current flow and analysis of the spontaneous baseline noise. All data are presented as mean \pm SEM. Statistical significance was determined using the Student's two-tailed paired $t$ test, except when comparing separate genotypes (nompC vs $c n$ $b w$ ). There, a Student's two-tailed unpaired equal variance $t$ test was used.

Hypoxia. Hypoxia was achieved by a flow of nitrogen over the fly for 2-3 min. A nitrogen flow rate of $35 \mathrm{ml} / \mathrm{s}$ through a tube $7 \mathrm{~mm}$ in diameter did not cause movement of bristles or other body parts. For control, air was blown over the fly for $2-3 \mathrm{~min}$.

Calcium imaging. Imaging of the anterior notopleural bristle in Cha-Gal4, UAS-GCaMP3 flies was performed with an Olympus AX70 microscope. The bristle was deflected using an insect pin mounted on a manual micromanipulator. Flies heterozygous for Cha-Gal4 and UAS-GCaMP3 were used.

Heating. For dTrpA1 experiments, a heating pad of a 1/16 DIN autotune controller (Omega Bioengineering) was placed $2 \mathrm{~mm}$ below the fly and the temperature sensor. Both electrophysiological as well as calcium imaging recordings were performed after $60 \mathrm{~s}$ heating to $32^{\circ} \mathrm{C}$. Recovery was determined after the temperature fell below $30^{\circ} \mathrm{C}$.

\section{Results}

Characterization of activation-induced long-term adaptation As a first step toward elucidating the long-term gain control mechanisms we characterized the lingering depression of test responses 
induced by mechanical activation. To this end, we measured current responses of single mechanoreceptor neurons to deflections of the anteronotopleural bristle (Walker et al., 2000). A consistent comparison between flies was obtained by normalizing the stimuli and response amplitudes to the values of saturating pulses (expressed as percentage of saturation, \%Sat). The extent of depression was determined by measuring the effect of a preceding conditioning stimulus on the response amplitude of a subsequent test-pulse.

To define the effect of activation we have determined the degree of depression and how long the effect lasts. We have determined these effects of preceding activation at four incrementing testpulse intensities. When the test-pulse was at a saturating intensity $(100 \%$ Sat) it was minimally depressed by the conditioning-pulse (Fig. $1 A$, top left trace). In contrast, a test pulse at $40 \%$ Sat was substantially depressed by a preceding $100 \%$ Sat conditioning-pulse (Fig. $1 A$, bottom left trace). The initial depression of the test response recovered when the interval between the two stimuli was longer (Fig. $1 A$, right vs left traces). A complete series of interstimulus intervals and test-pulse magnitudes revealed that depression induced by conditioning activation was specific to low-amplitude test pulses (Fig. $1 B$ ). Both the initial extent of depression and its persistence reached a maximum when the test pulses were $60 \%$ Sat and a further reduction to $40 \%$ Sat did not exacerbate the effect significantly (Fig. $1 B$ ). The marked depression of response amplitudes specifically at the lower end of stimulus intensity may represent a decrease in sensitivity. To establish that the preceding activation elicits a sensitivity reduction we determined the effect of a $100 \%$ Sat conditioning stimulus on the entire range of test responses. The current-displacement curve was right-shifted by $1.02 \pm 0.08 \mu \mathrm{m}$, accounting for the dramatic effect at the lower end of stimulus magnitudes (Fig. 1C). After activation, the slope of the current-displacement curve was also $1.74 \pm 0.18$ times shallower, while the maximal response amplitudes were not affected (Fig. 1C). Thus, activation of Drosophila mechanoreceptor neurons elicits a long-term adaptive process that diminishes sensitivity. During this long-term adaptation, a wider range of stimulus intensities can be encoded by the receptor neuron since its full response range does not change while the slope of the current-displacement curve is shallower (Fig. 1C).

After determining that activation elicits long-term adaptation we sought to determine how the duration of conditioning pulses affect test-response depression. This relationship reflects the time course of the cellular process that generates long-term adaptation during the conditioning activation. The long-term adaptation onset time course was determined by applying conditioning stimuli at incrementing durations. We found that the gradual onset of long-term adaptation had a time constant of $441 \pm 61 \mathrm{~ms}$ (Fig. 2 B). Therefore, long-term adaptation extent is determined by a summation of preceding activation through hundreds of milliseconds. Similar to the effect of duration, the amplitudes of the conditioning stimuli also correlated with the extent of testresponse depression (Fig. $2 C, D$ ). Since both the durations and
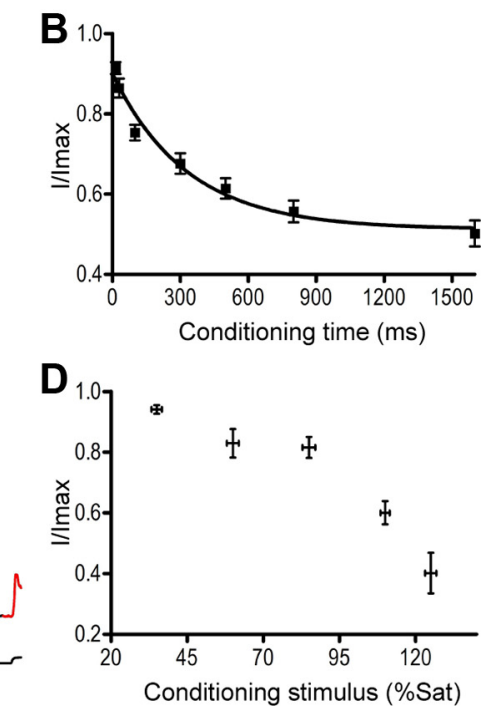

Figure 2. Effects of the duration and intensity of conditioning stimuli on test-responses. $A$, Depression induced by 30 and $1600 \mathrm{~ms}$ conditioning pulses on $50 \%$ Sat test pulses. $\boldsymbol{B}$, Effect of incrementing durations of conditioning pulses on $50 \%$ Sat test responses at a $300 \mathrm{~ms}$ incrementing intensities of conditioning pulses on 60\%Sat test responses at a 300 ms interval. Supersaturating stimuli (>100\%Sat) required nonlinearly stronger stimuli as demonstrated in top panel. For each data point, conditioning stimulus intensities were binned post-acquisition according to their percentage of saturation. $n \geq 4$. Error bars represent means \pm SEM.

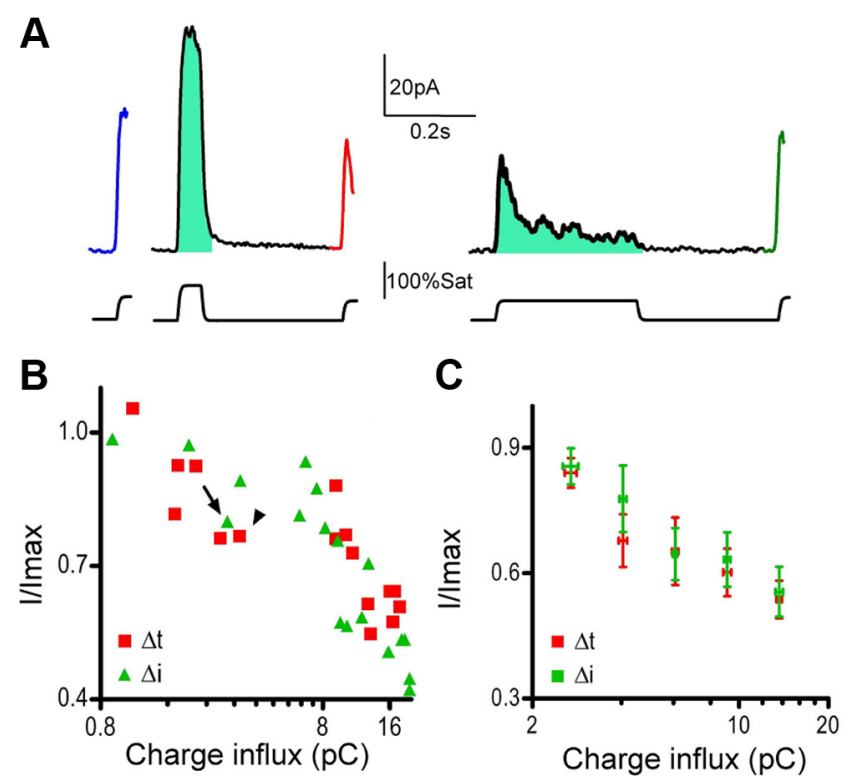

Figure 3. Effect of total charge influx during conditioning activation on test-responses. $\boldsymbol{A}$, The effect of a short and strong conditioning response is comparable to that of a weak and long pulse (arrowhead vs arrow in $\boldsymbol{B}$ ). The two responses had very similar total charge inflows, represented by the green area under the response trace. $\boldsymbol{B}$, An example of the consistent effect of charge on test response depression within one fly. The relation was comparable for changes in the duration $(\Delta t)$ and the intensity $(\Delta i)$ of the conditioning responses. $\Delta t: 15,30,50,100,200,300,500,800,1600,3200$ ms and $\Delta i$ : 15-125\%Sat, measured post-acquisition. C, A summary of the relationship between conditioning response charge and the depression of subsequent test stimuli. The calculated charge influx was binned in a power of 1.5 intervals. $n=8-13$. Error bars represent means \pm SEM.

intensities of conditioning stimuli strongly affected long-term adaptation, we postulated that cumulative response currents mediate the effect. This hypothesis predicts that the total charge carried into the cell during the conditioning response would correlate with the extent of long-term adaptation. To test the 
A
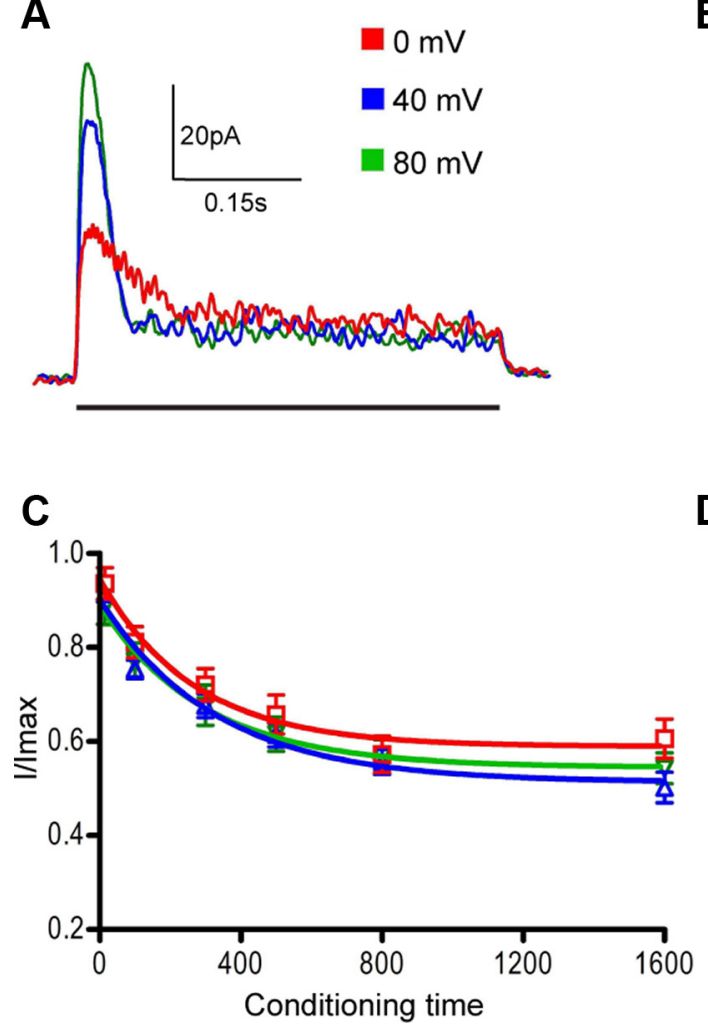

B

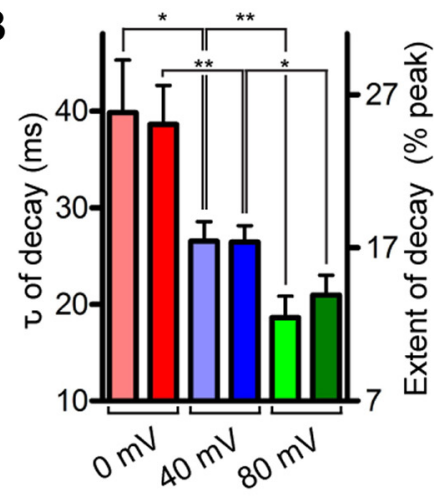

D

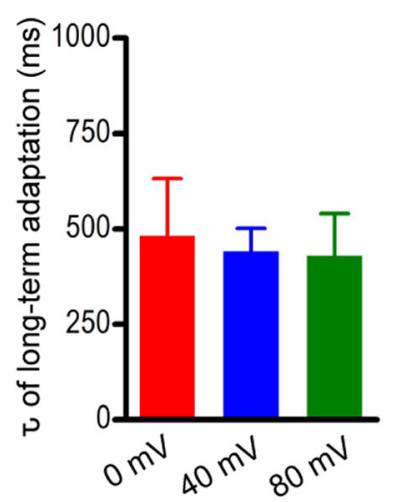

Figure 4. Long-term adaptation and adaptive response decay are independently modulated by the holding potential. $\boldsymbol{A}$, Responses at holding potentials of $80 \mathrm{mV}$ (green), $40 \mathrm{mV}$ (blue), and $0 \mathrm{mV}$ (red). B, Time courses (left axis, lighter shades) and extents (right axis, darker shades) of adaptive current decay under the different holding potentials. $n \geq 14$. ${ }^{*} p<0.02,{ }^{* *} p<0.01$. C, Generation of long-term adaptation at three different holding potentials. Experiments were performed as in Figure $2 B$, with $50 \%$ Sat test stimuli. $n \geq 11$. D, The time constant of long-term adaptation onset at the different holding potentials. $n \geq 12$. Error bars represent means \pm SEM.
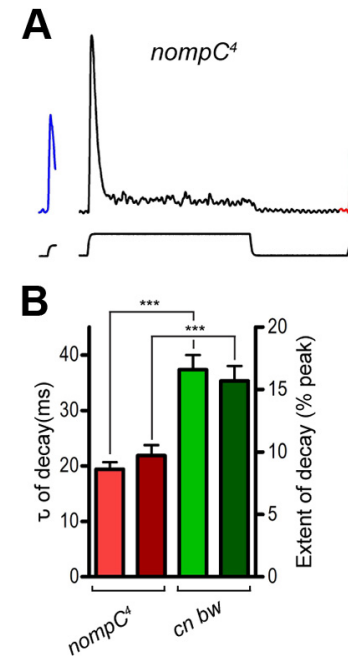

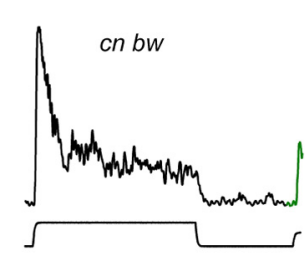

$\mathrm{C}_{10}$

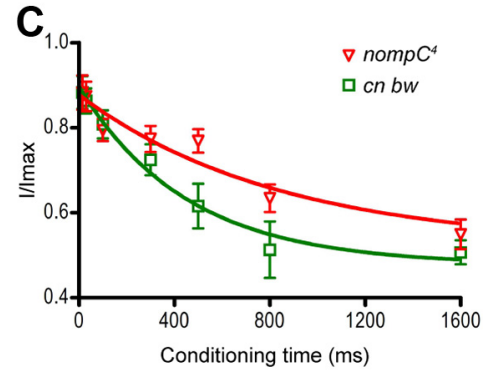

Figure 5. Long-term adaptation and adaptive response decay are independently modulated by the nomp $C^{4}$ mutation. $\boldsymbol{A}$, Depression by conditioning stimuli in nomp $C^{4}$ and control flies. $\boldsymbol{B}$, Time courses (left axis, lighter shades) and extents (right axis, darker shades) of adaptive current decay in nomp $C^{4}$ and control flies. $n \geq 17$. ${ }^{* * *} p<0.001$. C, Long-term adaptation onset in nomp $C^{4}$ and control flies. As controls we used $c n, b w$ flies, which are the genetic background of the nomp $C^{4}$ strain. $n \geq 5$. Error bars represent means \pm SEM.

prediction we measured the integrated currents elicited by the conditioning stimuli and plotted them against the resulting response depression (Fig. 3). Indeed, we found a strong correlation between the total charge carried into the mechanoreceptor

neuron during conditioning pulses and the depression of test-response (Fig. $3 B, C)$. Moreover, the same correlation was observed whether we changed the amplitudes or the durations of the conditioning stimuli (Fig. $3 B, C$ ). The consistent relationship between charge inflow and response depression suggests that long-term adaptation is caused by a gradual accumulation of ions that flow through the mechanically activated channels.

\section{Long-term adaptation and adaptive} response decay are distinct processes To explore how gain is suppressed in long-term adaptation we examined whether it is generated by the same adaptation process that rapidly reduces the gain during stimulation. A common cellular process underlying both the adaptive response decay and long-term adaptation would generate the two processes with the same time course, while separate transduction mechanisms would elicit independent kinetics. We found that even at the shortest interstimulus interval, the adapted phase of the response demonstrated a nearly complete recovery at $100 \%$ Sat test stimuli (Fig. $1 A$, top left trace). At the same interval the depression of weak stimuli by long-term adaptation was substantial (Fig. $1 A$, bottom left trace), suggesting that the recovery from adaptive response decay occurs at a faster time scale than long-term ad-
aptation. Accordingly, we found that the time constant of long-term adaptation onset (extracted from Fig. 2 B) was $>15$ times slower than the generation of adaptive response decay $(441 \pm 61$ vs $26 \pm 2 \mathrm{~ms})$.

The drastically different kinetics of the two processes supports the hypothesis that they constitute separate regulatory pathways. If indeed separate mechanisms mediate the two processes, they are also expected to be affected independently by manipulations of mechanotransduction. To test whether the processes are linked, we stimulated the bristle at different holding potentials, a treatment which is known to affect adaptation properties (Assad et al., 1989; Crawford et al., 1989). Changing the holding potentials had the expected effects on the extent and kinetics of adaptive response decay (Fig. 4A, B) while it had no impact on the properties of long-term adaptation (Fig. $4 C, D$ ). Thus, the two processes are independently affected by the electrochemical gradient. In an alternative approach to manipulating adaptation processes, we examined the mutant nomp $C^{4}$ which manifests abnormally rapid adaptive response decay (Walker et al., 2000). The adaptive response decay in the mutant was faster and stronger (Fig. $5 A, B$ ) while long-term adaptation had the opposite effect, developing slower and to a lesser extent (Fig. 5C). Therefore, a mutation in a specific transduction component independently affects adaptive response decay and long-term adaptation.

In summary, adaptive response decay and long-term adaptation operate in different time scales and through distinct transduction pathways. Nevertheless, the charge-mediated generation of long- 
term adaptation was not affected either by changing the holdingpotential (Fig. $6 A$ ) or by the nomp $C^{4}$ mutation (Fig. $6 B$ ). Long-term adaptation is therefore consistently related to charge inflow, even when adaptive response decay is significantly altered.

Long-term adaptation results from suppression of active amplification

Given that long-term adaptation is not associated with the adaptive response decay, we investigated the possible involvement of active mechanical amplification. Active amplification is characterized by nonlinear compression, which essentially entails that the gain is disproportionally higher at lower stimulus intensities (Hudspeth, 2008). Consequently, inhibition of active amplification would suppress the gain specifically at the lower range of test stimuli, as we observed in long-term adaptation (Fig. $1 \mathrm{~B}$ ). Therefore, it is possible that long-term adaptation is mediated by a suppression of active amplification. To further support this hypothesis we studied the spontaneous emission of electrical noise (Crawford and Fettiplace, 1985; Martin et al., 2003) which is proposed to be a manifestation of active amplification (Martin et al., 2003). A link between active amplification and long-term adaptation predicts that activation would have the same depressing effect on test responses and on the spontaneous noise. We quantified the effects of conditioning stimuli on the spontaneous noise and found a postresponse depression that gradually recovered to control levels (Fig. 7A, top trace). As in the effect of long-term adaptation on test responses, the time course of noise depression depended on the intensity and duration of the conditioning pulse (Fig. $7 A, B)$. Furthermore, the time constants of recovery from noise depression and from long-term adaptation were similar (Fig. 7C). Therefore, preceding activation elicits the same depressing effect on test responses and on spontaneously emitted noise.

An additional prominent feature of active mechanical amplification is the need for energy input (Hudspeth, 2008). Previous studies have shown that energy deprivation using hypoxia induces reversible reductions in both sensitivity (Robertson and Manley, 1974) and spontaneous noise emissions (Ohyama et al., 1991). To test whether inhibiting active amplification elicits long-term adaptation we curbed the amount of available energy using hypoxia. To quantify the effects of energy deprivation, the test responses and electrical noise were measured during a flow of nitrogen over the fly, and compared with pretreatment values. After the hypoxia treatment, we verified that reoxygenation restored both the sensitivity and the noise to verify that the treatment did not cause cellular damage.

Hypoxia reversibly depressed both the lower-range responses (Fig. $8 A, C$ ) and the emission of spontaneous noise (Fig. $8 B, D$ ). In addition, similar to the diminished sensitivity caused by conditioning stimuli (Fig. 1C), hypoxia treatment generated a right-shift in the
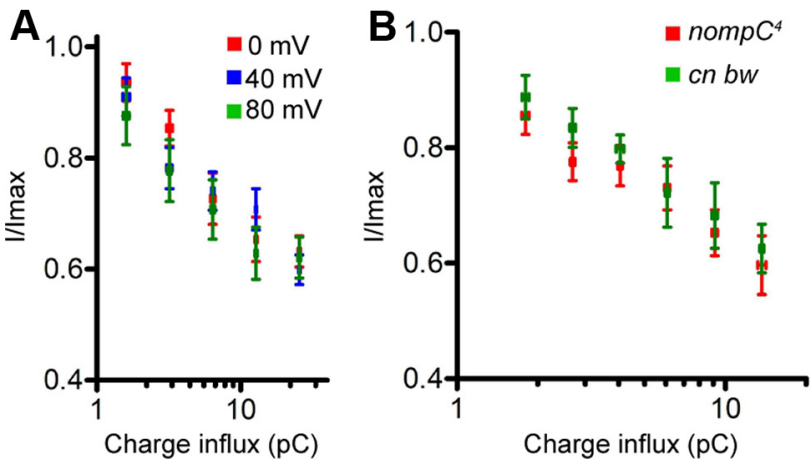

Figure 6. Consistent correlation between charge influx and the extent of long-term adaptation. $\boldsymbol{A}$, Relationship between test-response depression and integrated conditioning response at different holding potentials. $n=9-14$. $\boldsymbol{B}$, Relationship between test-response depression and integrated conditioning response in nomp $C^{4}$ and control $(c n, b w)$ flies. The calculated charge influx was binned in a power of 1.5 intervals. The data were pooled from the time course experiments ( $\boldsymbol{A}$ from Fig. 4C; $\boldsymbol{B}$ from Fig. $5 C$ ) with stimulus durations of (in ms) 15,100 , $300,500,800,1600 . n=9-18$. Error bars represent means \pm SEM.
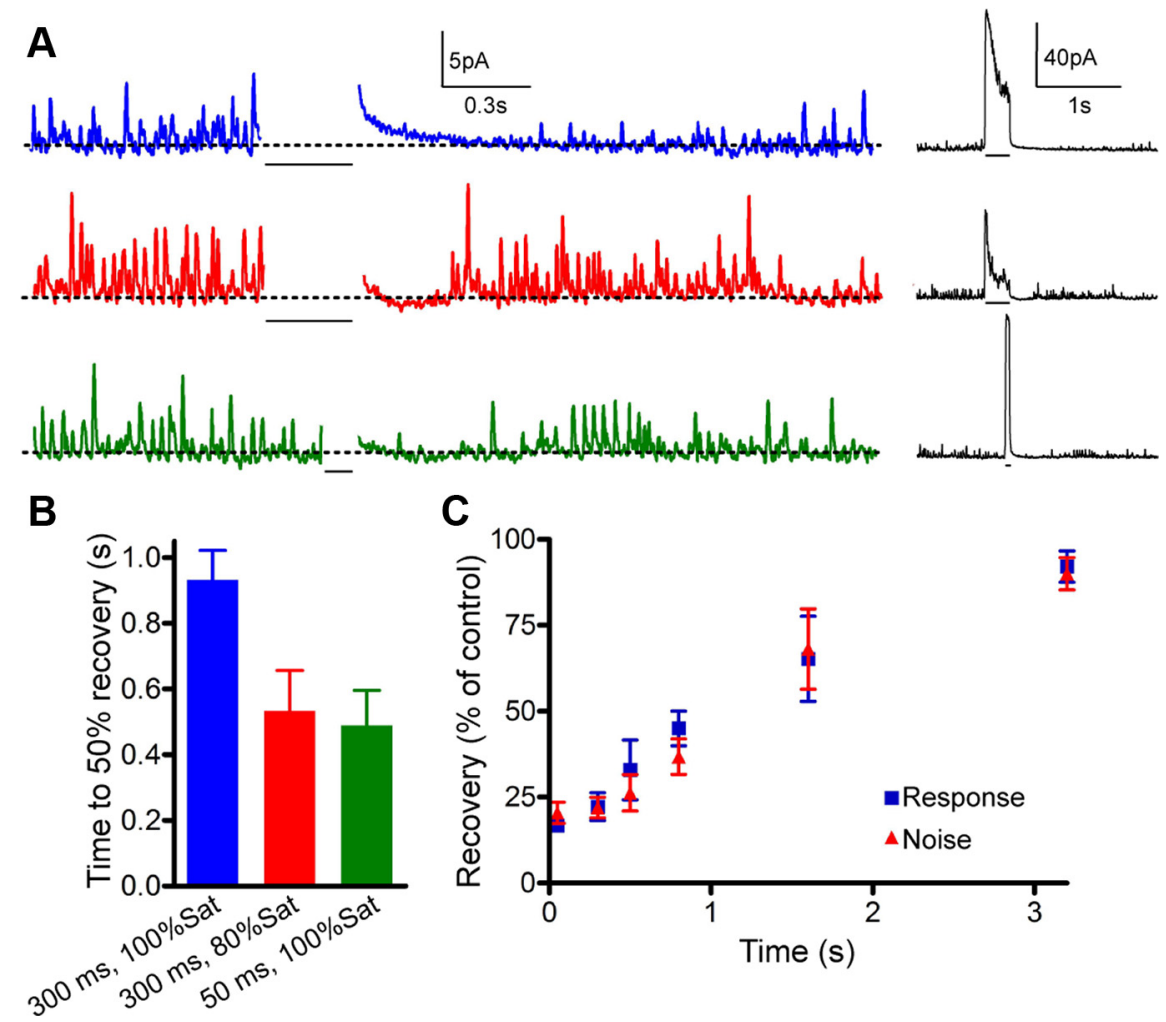

Figure 7. Activation depresses spontaneous noise. $\boldsymbol{A}$, Electrical noise is depressed by a $100 \%$ Sat, 300 ms stimulus (blue). Weaker (red, 70\%Sat) or shorter (green, $50 \mathrm{~ms}$ ) stimuli result in faster noise recovery. Insets show the same traces at a scale that demonstrates the full responses. $\boldsymbol{B}$, Average times to $50 \%$ recovery of the variance. $n=6-11$. $\boldsymbol{C}$, Recovery of noise and test responses from depression induced by conditioning activation. The depression of both noise and test responses were measured in the same flies. Test stimuli were $<50 \%$ Sat. $n=4-7$.

current-displacement curve (Fig. $8 E$ ). To ensure that the nitrogen flow did not cause any mechanical effects, we verified that the flow of air did not affect test responses (Fig. $8 F$ ). We further found that the contribution of hypoxia was additive to the induction of long-term adaptation by conditioning stimuli, resulting in a greater extent of long-term adaptation (Fig. 8G, maximal depression was $0.50 \pm 0.03$ in control and $0.17 \pm 0.04$ during hypoxia). Hypoxia had no effect on the kinetics of adaptive response decay ( $26 \pm 4 \mathrm{~ms}$ control and $26 \pm 6 \mathrm{~ms}$ hypoxia) or the maximal response amplitude (Fig. $8 E$ ), 
A

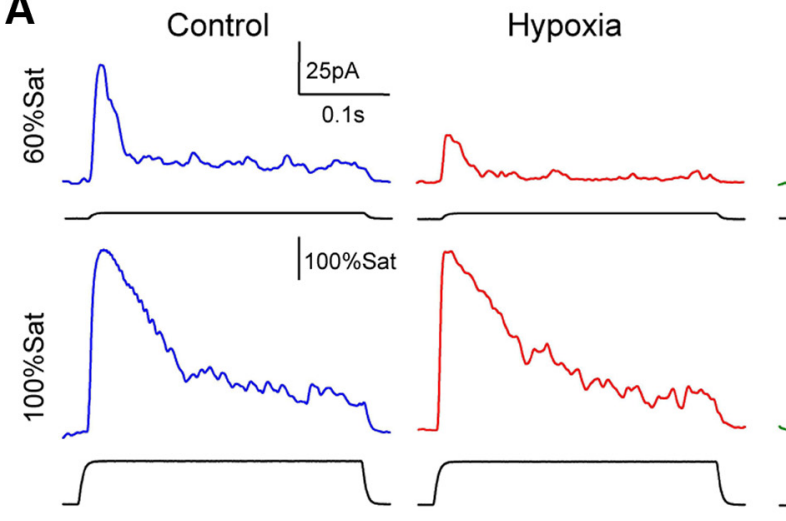

B

Control

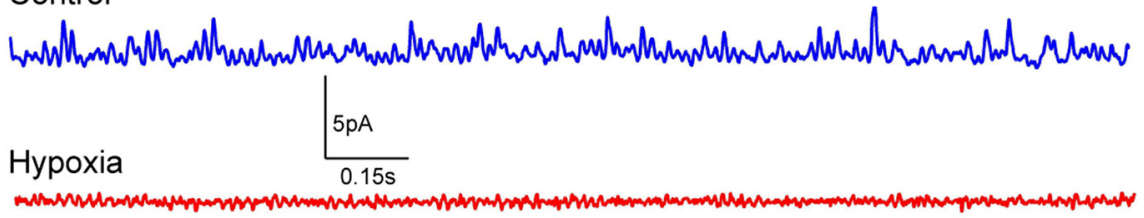

Recovery

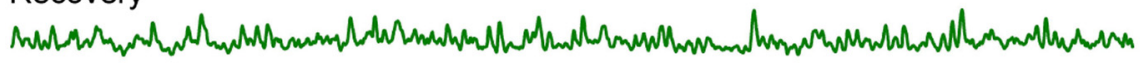

C

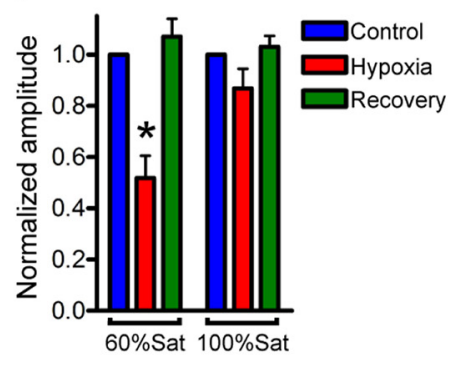

$\mathbf{F}$

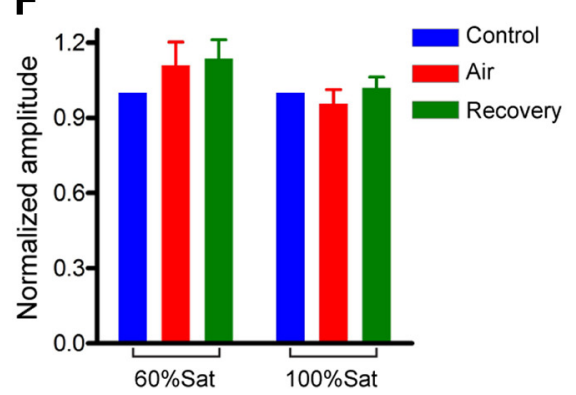

Recovery
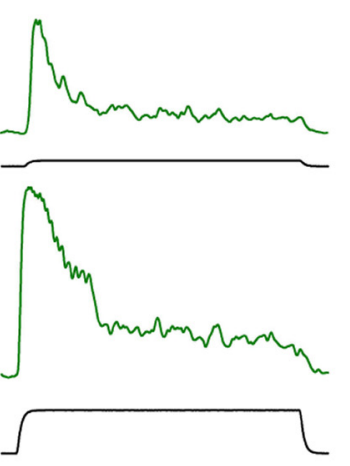

Generation of long-term adaptation correlates with cytoplasmic $\mathrm{Ca}^{2+}$ levels The correlation between the integrated conditioning currents and the extent of longterm adaptation (Figs. 3, 6) suggests that they are linked by the accumulation of one of the inflowing ions. Calcium ions are a major component of mechanically activated currents (Lumpkin et al., 1997) and they are known to modulate adaptation (Eatock et al., 1987; Ricci and Fettiplace, 1997). Therefore, $\mathrm{Ca}^{2+}$ accumulation may serve as the mediator between activation and long-term adaptation. If long-term adaptation is mediated by cytoplasmic $\mathrm{Ca}^{2+}$ levels then the kinetics of its onset should correlate with mechanoreceptor neuron $\mathrm{Ca}^{2+}$ accumulation while regaining sensitivity would match $\mathrm{Ca}^{2+}$ extrusion rate. To test $\mathrm{Ca}^{2+}$ dynamics during responses to mechanical stimuli we stimulated the anteronotopleural bristles while imaging the $\mathrm{Ca}^{2+}$-dependent fluorescence of mechanoreceptor neurons. The receptor neurons expressed the genetically encoded $\mathrm{Ca}^{2+}$ indicator GCaMP3 (Tian et al., 2009). Indeed, both the elevation in $\mathrm{Ca}^{2+}$ fluorescence during activation and its drop after cessation matched with the corresponding time courses of long-term adaptation (Fig. 9).

To further demonstrate the role of $\mathrm{Ca}^{2+}$ in long-term adaptation we directly manipulated its cellular concentration using the genetically encoded, heatactivated channel dTrpA1 (Hamada et al., 2008). It is expected that opening dTrpA1 channels would result in accumulation of $\mathrm{Ca}^{2+}$ and thereby mimic the effects of conditioning activation. First, we expressed dTrpA1 in fly mechanoreceptor neurons and verified that heating indeed elicited an elevation of cellular $\mathrm{Ca}^{2+}(\Delta F / F$ of $0.22 \pm 0.04$ in dTrpA1-expressing flies, $-0.12 \pm 0.02$ in controls). To quantify the effects of dTrpA1 activation, the test responses and electrical noise during a heating treatment were compared with pretreatment values. To ensure that the effects of heating were not a result of cellular damage we also verified that both the sensitivity and the spontaneous noise were restored after cooling. Heating depressed both the lower-range test responses and the spontaneous noise in dTrpA1-expressing flies; however, neurons of control flies were not affected

Figure 8. Hypoxia mimics the long-term depressing effects of activation. $\boldsymbol{A}$, Responses to test stimuli are shown before (blue), during (red) and after (green) hypoxia. $\boldsymbol{B}$, Spontaneous noise traces. C, Summary of hypoxia effects on $60 \%$ Sat and $100 \%$ Sat stimuli. $n=8$. $\boldsymbol{D}$, Summary of hypoxia-induced noise depression. Response and noise magnitudes were normalized to the prehypoxia values. $n=8$. $\boldsymbol{E}$, An example of current-displacement curves that were obtained before (blue) and during (red) hypoxia. Solid lines are Boltzmann fits and the dotted line is the extent of suppression. $\boldsymbol{F}$, Responses to test stimuli are shown before (blue), during (red) and after (green) the flow of air. Response magnitudes were normalized to the pre-flow values. $n=5$. $\mathbf{G}$, Generation of long-term adaptation by conditioning stimuli under control and hypoxic conditions. $n \geq 6$.

demonstrating that the mild energy deprivation we induced did not affect general response properties. Together, the effects of hypoxia demonstrate that a decrease in energy availability mimics the effects of conditioning pulses.
(Fig. 10). Thus, dTrpA1 activation was sufficient for recapitulating long-term adaptation. The correlation between longterm adaptation kinetics and cellular $\mathrm{Ca}^{2+}$ dynamics, together with the depressing effect of $\mathrm{dTrpA} 1$ activation, suggest that 
A

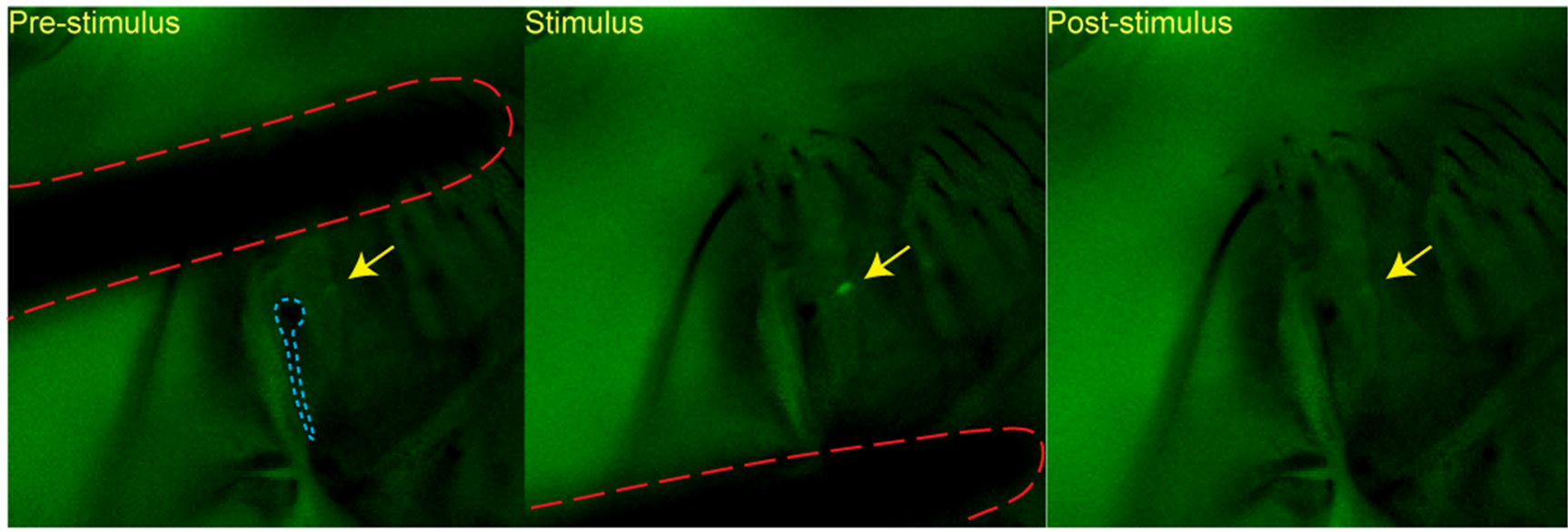

B

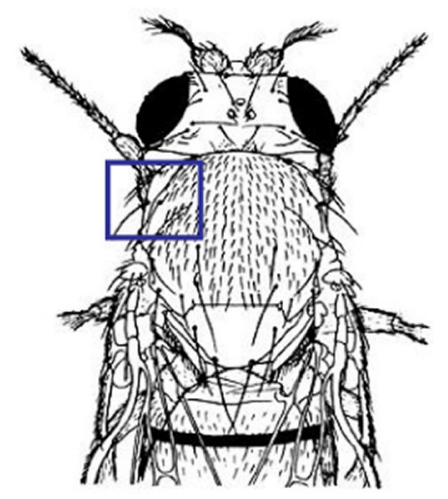

C

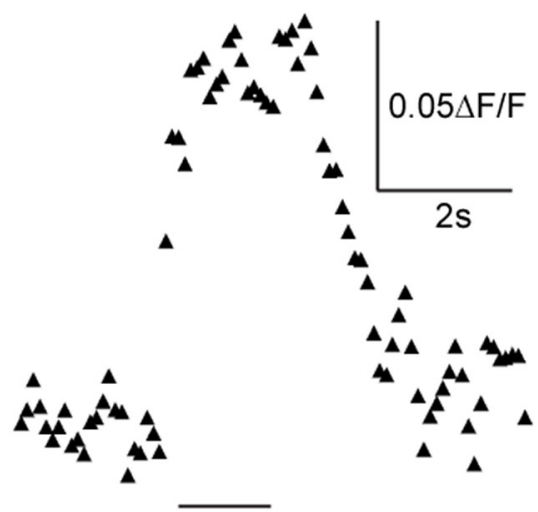

D

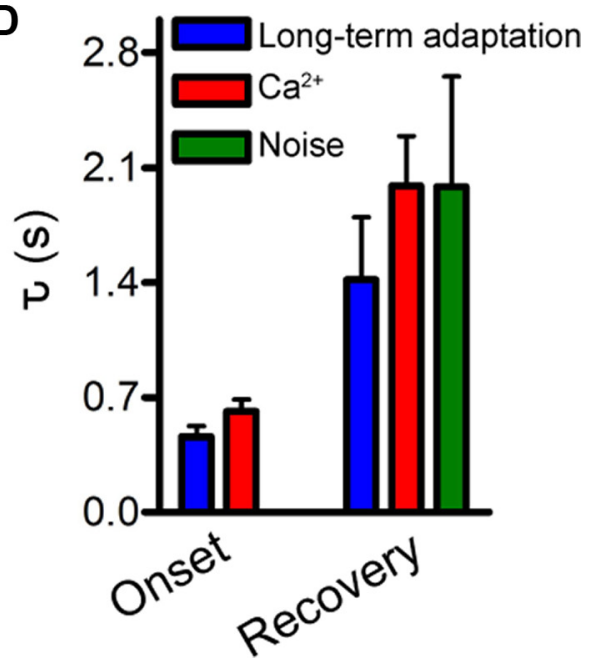

Figure 9. Time courses of $\mathrm{Ca}^{2+}$ response match long-term adaptation kinetics. $A$, In vivo GCaMP3 fluorescence imaging from the mechanoreceptor neuron of the anteronotopleural bristle. Images are taken at the focal plane of the cell body (arrow) so that the bristle (dashed blue) and the stimulating probe (dashed red) are slightly out of focus. B, Schematic drawing showing the imaged region. $C$, A typical $\mathrm{Ca}^{2+}$ fluorescence response. $\boldsymbol{D}$, The average rates of $\mathrm{Ca}^{2+}$ increase are compared with the onset of long-term adaptation (Onset) and the average rates of $\mathrm{Ca}^{2+}$ decline are compared with the recovery of spontaneous noise and the recovery of test response amplitudes (Recovery). $n=6-11$.

accumulation of cytoplasmic $\mathrm{Ca}^{2+}$ leads to the generation of long-term adaptation.

\section{Discussion}

Characterization of activation-induced long-term adaptation We have found that following activation the gain in mechanoreceptor neurons is depressed for hundreds of milliseconds and that this depressing effect is generated over a similar time scale (Figs. 1, 2). These characteristics are in accord with findings in other mechanoreceptor neurons (Hao and Delmas, 2010; Kang et al., 2010; Rugiero et al., 2010). The depressing effect of activation was substantial at the lower end of test-response intensities and minimally affected responses at saturation (Fig. $1 B$ ). Therefore, the temporal resolution of mechanotransduction remains high when incoming signals are at saturating levels but severely diminishes when weak stimuli follow strong ones.

Changing the duration and the magnitude of conditioning pulses had comparable effects on long-term adaptation (Fig. 2 ). Since we observed a consistent correlation between the extent of long-term adaptation and the integrated conditioning current (Figs. 3,6) we postulate that the reduction of gain is determined mainly by integrating activation during a wide time window.

The current-displacement curve during long-term adaptation was characterized by a right-shift along the displacement axis, accompanied by a reduction in slope (Fig. $1 C$ ). A right-shift in the current-displacement curve is a well described characteristic of adaptation and it typically retains the slope of the curve (Eatock et al., 1987; Hacohen et al., 1989). Nevertheless, reductions in slope have also been reported in hair cells, mainly by application of large adapting steps (Assad and Corey, 1992; Holt et al., 1997; Vollrath and Eatock, 2003). Therefore, it is possible that long-term adaptation and slow adaptation share underlying mechanisms.

\section{Long-term adaptation and adaptive response decay are distinct processes}

To identify the transduction process that underlies long-term adaptation we tested the possibility that it is generated by the same mechanism that causes the adaptive response decay. We found that upon stimulation with two consecutive saturating stimuli the response recovery was nearly complete even at the 
A

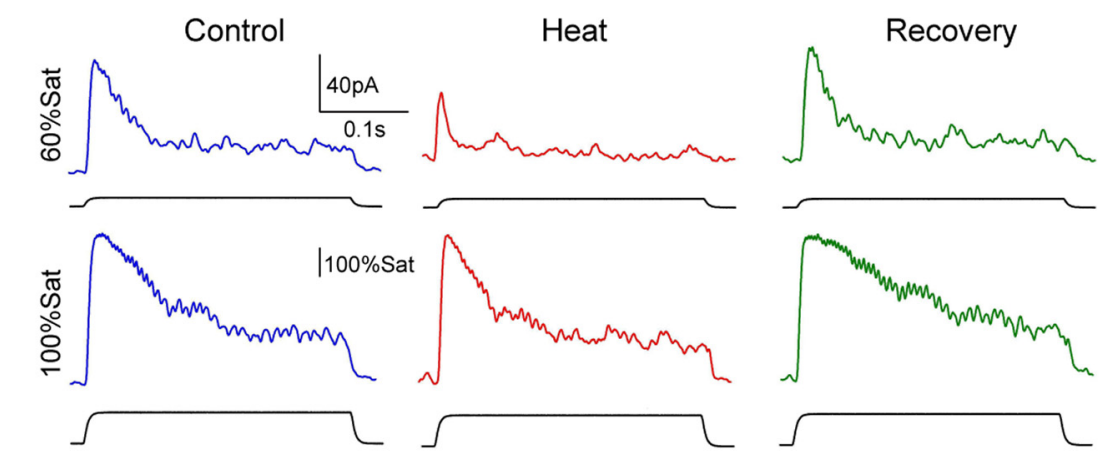

B

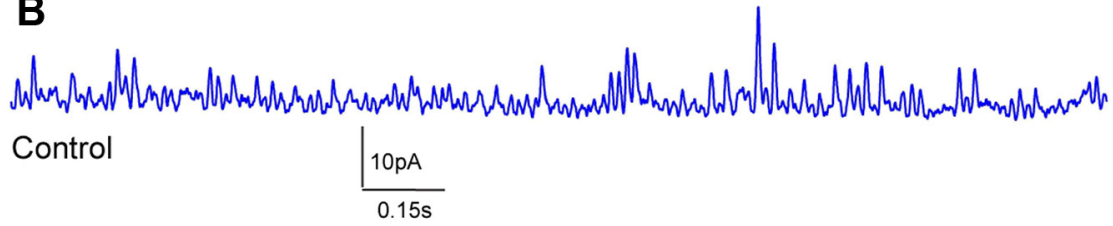

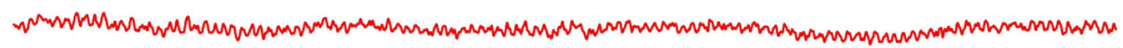
Heat

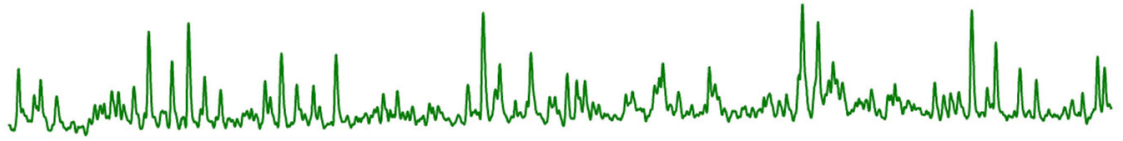

Recovery

C
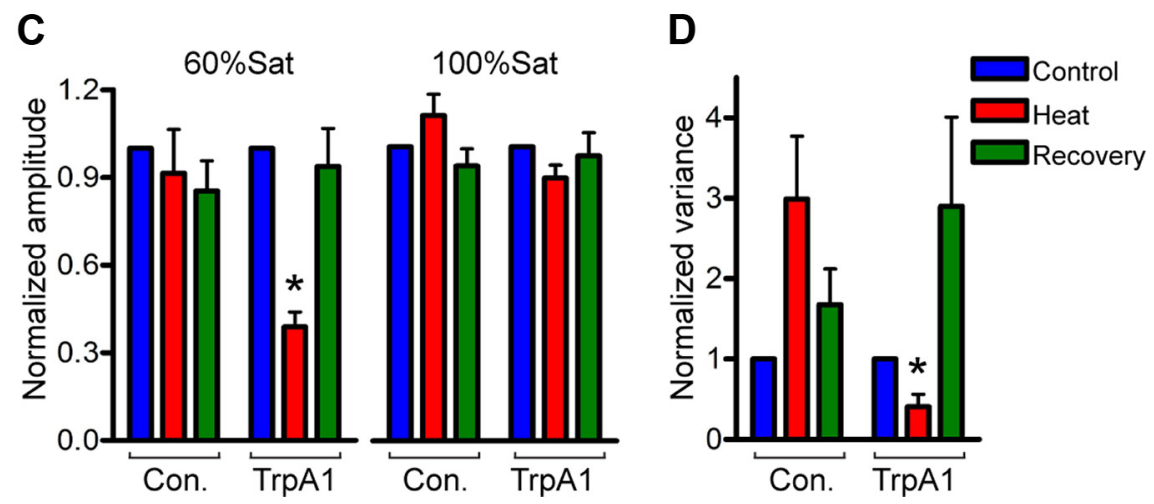

Figure 10. dTrpa1 activation mimics the long-term depressing effects of activation. $\boldsymbol{A}$, Current responses in a dTrpA1expressing fly are shown before (blue), during (red) and after (green) heating. B, Representative spontaneous noise traces. C, Summary of heat-induced response depression in control (Con.) and dTrpA1-expressing flies. Responses at $60 \%$ Sat and $100 \%$ Sat are compared. $n=7-11 . D$, Summary of heat-induced noise depression. Response and noise magnitudes were normalized to the pre-heating values. $n=6 .{ }^{*} p<0.02$. Error bars represent means \pm SEM.

shortest interval (Fig. $1 A$, top traces). The mechanoreceptor neuron's ability to evoke the transient phase shortly after the termination of a fully adapted response suggests that the adaptive response decay reverses rapidly. In addition, the generation of long-term adaptation and adaptive response decay take place at very different time scales; when adaptive response decay was already at a steady-state level, the effect of long-term adaptation continued to grow considerably (Fig. $2 \mathrm{~B}$ ).

Given that adaptive response decay saturates long before longterm adaptation, it is likely that the two processes are generated in parallel. To further test the link between long-term adaptation and adaptive response decay we changed the holding potential, a manipulation that substantially affects adaptive response decay (Assad et al., 1989; Crawford et al., 1989). We found that the changes in the properties of adaptive response decay were not associated with an effect on long-term adaptation (Fig. 4). To characterize the process at the molecular level we tested a specific mutation that affects adaptation in nompC (Walker et al., 2000), which is a mechanosensitive channel (Cheng et al., 2010; Kang et al., 2010). The mutant, nomp $C^{4}$, demonstrated the expected rapid kinetics of adaptive response decay; however, they were not associated with corresponding changes to long-term adaptation (Fig. 5). Therefore, both by changing the holding potential and by a specific mutation we demonstrated that changes in the adaptive response decay are not accompanied by corresponding changes in long-term adaptation. The lack of association was also supported by the converse observation; induction of long-term adaptation using hypoxia was not accompanied by an effect on adaptive response decay (Fig. 8).

Altogether, our findings indicate that there are at least two separate gain adjustment processes in Drosophila mechanotransduction: the rapid decay of response amplitude during a stimulus and long-term adaptation, which adjusts mechanotransduction sensitivity to the average stimulus intensity over a wide time window. Previous studies in hair cells demonstrated that adaptation to sustained stimuli is comprised of two distinct processes with separate time courses (Wu et al., 1999). The time course of the slow adaptation process may reach hundreds of milliseconds (Eatock et al., 1987) suggesting that it may share a common underlying mechanism with the long-term adaptation that we observed. It has been suggested that by determining the set point of mechanotransduction, adaptation biases it toward operating at a region mechanical instability (Martin et al., 2000). The interplay between adaptation adjustment mechanisms and the point of instability is thought to underlie active amplification (Martin et al., 2000). It is therefore possible that the long-term adaptation results from shifting away from the region of mechanical instability, thereby diminishing amplification and spontaneous noise. Alternatively, since the time constant of long-term adaptation is still slightly higher than the upper range of slow adaptation, it is possible that a separate transduction mechanism is involved. Future studies are necessary for testing whether the slow component of response decay and the long-term adaptation are linked.

\section{Active amplification suppression and long-term adaptation}

The specific effect of long-term adaptation on low-end response intensities (Fig. $1 B$ ) and on the slope of the current-displacement curve (Fig. 1C) are in accord with reduced amplification. We discovered that a prominent feature of active amplification, the spontaneously emitted noise (Martin et al., 2003) is affected by conditioning activation in the same manner as test-responses (Fig. 7C). To further link between long-term adaptation and active amplification suppression we limited energy availability. 
Limiting energy availability using hypoxia has been shown to severely and reversibly diminish auditory sensitivity (Robertson and Manley, 1974) and the occurrence of spontaneous noise emissions (Ohyama et al., 1991). We have defined hypoxic conditions that did not affect general response characteristics (Fig. $8 E$ ) while they were sufficient for recapitulating long-term adaptation. The effects of hypoxia and preactivation were similar on both test-responses and the electrical noise (Fig. 8C,D), although the right-shift in the current-displacement curve was stronger during hypoxia. The stronger hypoxia effect is probably caused by the direct superposition of test pulses onto the treatment, as opposed to the partial recovery that occurs after the cessation of conditioning stimuli. In summary, there are three independent lines of evidence which indicate that long-term adaptation is mediated by active amplification suppression: preceding activation specifically affects sensitivity, activation diminishes the emission of spontaneous noise and both these effects can be mimicked by energy deprivation.

Active amplification requires energy and generates noise and it would therefore be evolutionarily advantageous to attenuate the gain during extended periods of elevated activation. Under such conditions amplification is rendered unnecessary since the elevated background levels would mask weaker incoming stimuli. Another beneficial feature of long-term adaptation arises from the mechanism of its induction which does not require external application of mechanical forces (Figs. 8, 10). This type of a mechanism, which probably involves a diffusible second messenger, is able to regulate sensitivity by integrating activation levels with additional cell-signaling factors. These factors may be intracellular, such as a reaction to cellular stress, or intercellular, as in hormonal signaling. Effects of cellular messengers on mechanotransduction properties have been reported for the cAMP (Ricci and Fettiplace, 1997; Martin et al., 2003) and PIP2 (Hirono et al., 2004) pathways.

\section{Cellular $\mathrm{Ca}^{2+}$ accumulation and long-term adaptation}

Calcium ions may be general modulators of mechanotransduction since they can modulate spontaneous mechanical oscillations (Martin et al., 2003) and they are necessary for both slow and fast adaptation (Wu et al., 1999). Therefore, the inflow of $\mathrm{Ca}^{2+}$ may underlie the consistent correlation between the total charge inflow during activation and the extent of long-term adaptation. To study $\mathrm{Ca}^{2+}$ dynamics during mechanical responses we developed a method for $\mathrm{Ca}^{2+}$ imaging from the same neurons that were characterized electrophysiologically (Fig. 9). We found that the dynamics of cellular $\mathrm{Ca}^{2+}$ levels were correlated with the time courses of long-term adaptation and dramatically slower than the kinetics of adaptive response decay (Fig. 9D). To induce cellular $\mathrm{Ca}^{2+}$ elevation we expressed the heat-activated channel dTrpA 1 in the mechanoreceptor neurons. We found that dTrpA1 activation was sufficient for recapitulating the effects of longterm adaptation (Fig. 10C,D). We propose that activation is integrated through the accumulation of $\mathrm{Ca}^{2+}$, a process which leads to suppression of active amplification through a yet unknown mechanism.

\section{Gain control mechanisms in Drosophila mechanotransduction}

Measurements of antennal motions and compound action potentials indicate that the auditory system of the fly actively amplifies mechanical stimuli (Göpfert et al., 2006) and adapts to them (Albert et al., 2007). Such studies are well suited for defining the contribution of molecular components to ampli- fication processes, because of the sensitive measurements of mechanical motions (Albert et al., 2007). However, these experiments measure the function of the entire hearing organ and are therefore unable to resolve response from single cells and to define mechanically induced currents. Here we were able to separate between distinct transduction processes and characterize their properties using measurements of current responses from single receptor neurons. Together, the sensitive motion analysis of amplification and detailed characterization of single cell responses are expected to be a useful platform for genetic studies of transduction components.

\section{References}

Albert JT, Nadrowski B, Göpfert MC (2007) Mechanical signatures of transducer gating in the Drosophila ear. Curr Biol 17:1000-1006.

Assad JA, Corey DP (1992) An active motor model for adaptation by vertebrate hair cells. J Neurosci 12:3291-3309.

Assad JA, Hacohen N, Corey DP (1989) Voltage dependence of adaptation and active bundle movement in bullfrog saccular hair cells. Proc Natl Acad Sci U S A 86:2918-2922.

Beurg M, Fettiplace R, Nam JH, Ricci AJ (2009) Localization of inner hair cell mechanotransducer channels using high-speed calcium imaging. Nat Neurosci 12:553-558.

Brass D, Kemp DT (1993) Analyses of Mossbauer mechanical measurements indicate that the cochlea is mechanically active. J Acoust Soc Am 93:1502-1515.

Cheng LE, Song W, Looger LL, Jan LY, Jan YN (2010) The role of the TRP channel NompC in Drosophila larval and adult locomotion. Neuron 67:373-380.

Crawford AC, Fettiplace R (1985) The mechanical properties of ciliary bundles of turtle cochlear hair cells. J Physiol 364:359-379.

Crawford AC, Evans MG, Fettiplace R (1989) Activation and adaptation of transducer currents in turtle hair cells. J Physiol 419:405-434.

Crawford AC, Evans MG, Fettiplace R (1991) The actions of calcium on the mechano-electrical transducer current of turtle hair cells. J Physiol 434:369-398.

De Vries HL (1948) Brownian movement and hearing. Physica 14:48-60.

Eatock RA, Corey DP, Hudspeth AJ (1987) Adaptation of mechanoelectrical transduction in hair cells of the bullfrog's sacculus. J Neurosci 7:2821-2836.

Gillespie PG, Müller U (2009) Mechanotransduction by hair cells: models, molecules, and mechanisms. Cell 139:33-44.

Göpfert MC, Humphris AD, Albert JT, Robert D, Hendrich O (2005) Power gain exhibited by motile mechanosensory neurons in Drosophila ears. Proc Natl Acad Sci U S A 102:325-330.

Göpfert MC, Albert JT, Nadrowski B, Kamikouchi A (2006) Specification of auditory sensitivity by Drosophila TRP channels. Nat Neurosci 9:999-1000.

Hacohen N, Assad JA, Smith WJ, Corey DP (1989) Regulation of tension on hair-cell transduction channels: displacement and calcium dependence. J Neurosci 9:3988-3997.

Hamada FN, Rosenzweig M, Kang K, Pulver SR, Ghezzi A, Jegla TJ, Garrity PA (2008) An internal thermal sensor controlling temperature preference in Drosophila. Nature 454:217-220.

Hao J, Delmas P (2010) Multiple desensitization mechanisms of mechanotransducer channels shape firing of mechanosensory neurons. J Neurosci 30:13384-13395.

Hirono M, Denis CS, Richardson GP, Gillespie PG (2004) Hair cells require phosphatidylinositol 4,5-bisphosphate for mechanical transduction and adaptation. Neuron 44:309-320.

Holt JR, Corey DP, Eatock RA (1997) Mechanoelectrical transduction and adaptation in hair cells of the mouse utricle, a low-frequency vestibular organ. J Neurosci 17:8739-8748.

Holt JR, Gillespie SK, Provance DW, Shah K, Shokat KM, Corey DP, Mercer JA, Gillespie PG (2002) A chemical-genetic strategy implicates myosin-1c in adaptation by hair cells. Cell 108:371-381.

Howard J, Bechstedt S (2004) Hypothesis: a helix of ankyrin repeats of the NOMPC-TRP ion channel is the gating spring of mechanoreceptors. Curr Biol 14:R224-R226.

Howard J, Hudspeth AJ (1987) Mechanical relaxation of the hair bundle mediates adaptation in mechanoelectrical transduction by the bullfrog's saccular hair cell. Proc Natl Acad Sci U S A 84:3064-3068. 
Hudspeth AJ (2008) Making an effort to listen: mechanical amplification in the ear. Neuron 59:530-545.

Hudspeth AJ, Gillespie PG (1994) Pulling springs to tune transduction: adaptation by hair cells. Neuron 12:1-9.

Jaramillo F, Markin VS, Hudspeth AJ (1993) Auditory illusions and the single hair cell. Nature 364:527-529.

Kang L, Gao J, Schafer WR, Xie Z, Xu XZ (2010) C. elegans TRP family protein TRP-4 is a pore-forming subunit of a native mechanotransduction channel. Neuron 67:381-391.

Kennedy HJ, Evans MG, Crawford AC, Fettiplace R (2003) Fast adaptation of mechanoelectrical transducer channels in mammalian cochlear hair cells. Nat Neurosci 6:832-836.

Lumpkin EA, Marquis RE, Hudspeth AJ (1997) The selectivity of the hair cell's mechanoelectrical-transduction channel promotes $\mathrm{Ca}^{2+}$ flux at low $\mathrm{Ca}^{2+}$ concentrations. Proc Natl Acad Sci U S A 94:10997-11002.

Martin P, Hudspeth AJ (1999) Active hair-bundle movements can amplify a hair cell's response to oscillatory mechanical stimuli. Proc Natl Acad Sci U S A 96:14306-14311.

Martin P, Hudspeth AJ (2001) Compressive nonlinearity in the hair bundle's active response to mechanical stimulation. Proc Natl Acad Sci U S A 98:14386-14391.

Martin P, Mehta AD, Hudspeth AJ (2000) Negative hair-bundle stiffness betrays a mechanism for mechanical amplification by the hair cell. Proc Natl Acad Sci U S A 97:12026-12031.

Martin P, Bozovic D, Choe Y, Hudspeth AJ (2003) Spontaneous oscillation by hair bundles of the bullfrog's sacculus. J Neurosci 23:4533-4548.

O'Hagan R, Chalfie M, Goodman MB (2005) The MEC-4 DEG/ENaC channel of Caenorhabditis elegans touch receptor neurons transduces mechanical signals. Nat Neurosci 8:43-50.

Ohyama K, Wada H, Kobayashi T, Takasaka T (1991) Spontaneous otoacoustic emissions in the guinea pig. Hear Res 56:111-121.

Ricci AJ, Fettiplace R (1997) The effects of calcium buffering and cyclic AMP on mechano-electrical transduction in turtle auditory hair cells. J Physiol 501:111-124.

Robertson D, Manley GA (1974) Manipulation of frequency analysis in the cochlear ganglion of the guinea pig. J Comp Physiol A Neuroethol Sens Neural Behav Physiol 91:363-375.

Rugiero F, Drew LJ, Wood JN (2010) Kinetic properties of mechanically activated currents in spinal sensory neurons. J Physiol 588:301-314.

Stauffer EA, Scarborough JD, Hirono M, Miller ED, Shah K, Mercer JA, Holt JR, Gillespie PG (2005) Fast adaptation in vestibular hair cells requires myosin-1c activity. Neuron 47:541-553.

Tian L, Hires SA, Mao T, Huber D, Chiappe ME, Chalasani SH, Petreanu L, Akerboom J, McKinney SA, Schreiter ER, Bargmann CI, Jayaraman V, Svoboda K, Looger LL (2009) Imaging neural activity in worms, flies and mice with improved GCaMP calcium indicators. Nat Methods 6:875-881.

Vollrath MA, Eatock RA (2003) Time course and extent of mechanotransducer adaptation in mouse utricular hair cells: comparison with frog saccular hair cells. J Neurophysiol 90:2676-2689.

Walker RG, Willingham AT, Zuker CS (2000) A Drosophila mechanosensory transduction channel. Science 287:2229-2234.

Wu YC, Ricci AJ, Fettiplace R (1999) Two components of transducer adaptation in auditory hair cells. J Neurophysiol 82:2171-2181. 